\title{
DesCom: Routing Decision using Estimation Time in VDTN
}

\author{
Adnan $\mathrm{Ali}^{1}$, Jinlong $\mathrm{Li}^{2}$, Aqsa Tanveer ${ }^{3}$, Maryam Batool ${ }^{4}$, Nimra Choudhary ${ }^{5}$ \\ School of Computer Science and Technology, University of Science and Technology China, Hefei, Anhui, China ${ }^{1,2}$ \\ Department of Computing and Information Technology, University of Gujrat, Sialkot, Pakistan ${ }^{3,4,5}$
}

\begin{abstract}
VDTN was proposed as a disrupting network which is established on the paradigm of the delay-tolerant network. VDTN uses vehicular nodes to convey messages as, it permits sparse opportunistic network connectivity, which is considered by the low node density where the vehicular traffic is sporadic, and no end-to-end paths exist between nodes. The message bundle is directed from the sender to the receiver node based on the routing protocol decision. While Routing protocols take decisions based on different metrics like Time to live, Location, Remaining Buffer Size, meeting probability, etc. In this paper, a routing protocol named DesCom is proposed for Vehicular Delay-Tolerant Network under a highly suppressed and sparse environment. DesCom takes the decision based on Message TTL, Transmission rate, and Estimation time. Estimation time is calculated in our previous work. The protocol defines whether to direct the message to the requested node or search the other more suitable node to carry that data bundles. After compiling multiple simulations with different numbers of vehicles and comparing DesCom with other routing protocols it is concluded that DesCom has the least buffer time with low latency along with good delivery probability.
\end{abstract}

Keywords-Estimation time; VDTN; routing; vehicular delaytolerant network; ONE simulator

\section{INTRODUCTION}

The vehicular ad-hoc network (VANET) [1] is the enhancement of the Mobile ad-hoc network with some distinctive characteristics. VANET is known for high mobility which leads to discontinuous communication to the areas consisting of low traffic and congestion in the area of high traffic. These factors disturb the performance of the network. To overcome these difficulties, the vehicular network may carry messages using the store, carry and forward paradigms of DTN as shown in Fig. 1 and, its extension VDTN is introduced. The idea of Delay Tolerant Networks[2] is to dig out from Interplanetary Network (IPN) which was started in 1970 to establish a communication between two planets (mars and earth). DTN consists of a message-oriented layer called "Bundle Layer" that is deliberate for unstable communication.

Datagrams (IP packets) are accumulated in wavering length data packets, termed as Bundles. The source node generates a data bundle and stores its information until it encounters another vehicular node. On meeting with the node, the message is transferred to the receiving nodes and it keeps happening until its TTL (Time to Live) [3] is expired.

Globally, smart cities are immoderately increasing day by day [4] due to the advancement of technology. Vehicular communication is essential for the smooth working of cities. The vehicular delay-tolerant network plays a significant role in communication where the network is not much dense. In a real-time scenario, each vehicle travels with different speed with unpredictable movement. Speed of vehicles vary due to the following scenarios [5]:

1) The road at which vehicle travel may be indented.

2) A throng of traffic inside the city is different from outside the city.

3) Highway speed is different from rural roads.

4) The type of vehicle which carried out the material may be different.

5) Timing is another factor in daylight, the speed of the vehicle is different from the night.

This paper derives in the sequence of previous work[5][6] in which authors explain connection time and calculate estimated time respectively. Estimation Time is a predictable time and calculated based on vehicle speed and distance (covered by a specific vehicle). Instead, Connection Time is the time between connection establishment and termination. The simulation results indicate that DesCom is better than some compared routing protocol of VDTN as it has the lowest buffer time with low average latency having a high delivery rate as compared to epidemic, prophet and direct delivery. This shows that the routing decision taken based on estimation time may lead to better decision making.

The remainder of this paper is organized according to the following sequence: Section I Introduction, Section II Vehicular delay Tolerant Network, Section III Literature Review, Section IV Methodology, Section V Results and Discussion, Section VI Conclusion and Future Work, and Section VII Acknowledgment. 


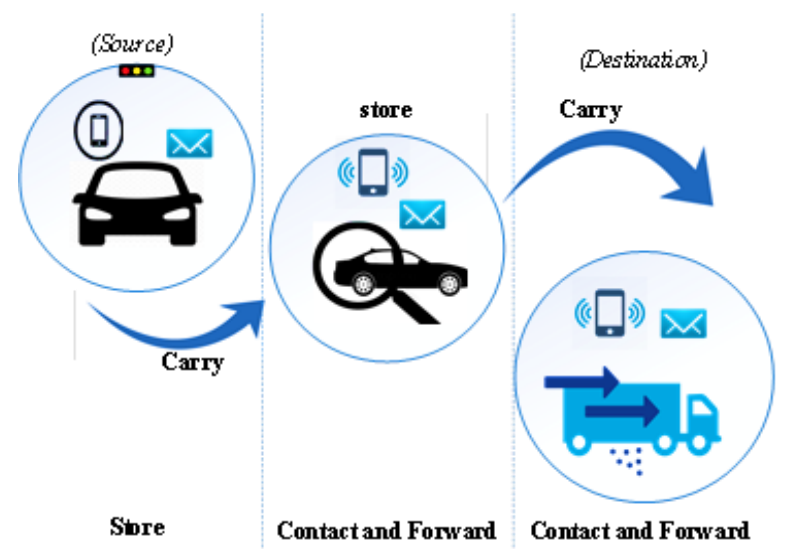

Fig. 1. DTN Paradigm.

\section{VEHICULAR DELAY-TOLERANT NETWORK}

DTN addresses the technical challenges which exist during communication between different devices as it loses its continuous connectivity due to high mobility. This network continues to advance the data even when from the source node to the destination node there is no complete identifiable path. DTN consists of a unique feature of intermittent connectivity and opportunistic communication [7] in which each node communicates with each other in the predefined time. The architecture of DTN was extended to transit networks, called Vehicular DTN or simply a VDTN. VDTN supports the concept of VANET with the Delay-Tolerant Network concept in network connectivity to support long disruption. It extends the capabilities of the delay-tolerant network for the vehicular environment [8]. VDTN is the extension of DTN in which vehicles interconnect with each other and with stationary nodes in order to place the message to the destination nodes travel along the road [9]. Most of the problem in the vehicular network is due to mobility as the speed of the vehicle as each vehicle travel with variant speed. VDTN is composed of mobile nodes that are wirelessly interconnected to one another despite connectivity issues. VDTN is growing the challenging field of DTN containing mobile nodes called vehicles. The idea behind this theory is that the node store message called bundle in its buffer until they find the opportunity to handover it to its destination or relay node [10]. The layered architecture of VDTN was initiated in [11] as illustrated in Fig. 2.

The bundle layer is specified as a message-oriented overlay layer in delay tolerant network as well as in vehicular delay-tolerant network. In DTN bundle layer occurs beyond the transport layers. Application data units are converted into protocol data units at the bundle layer and then data units are called "bundles" which are sent by DTN nodes according to the Bundle Protocol. The Bundle layer comprises a node-tonode transference of message having the responsibility for reliable delivery. In VDTN instead of positioning beyond the transport layer, the Bundle Layer is positioned inferior to the network layer because of communication problems like high error rates, high latency, long delay, sparse and discontinuous connectivity, and even end-to-end connectivity doesn't exist. The core objective is to convey the vast message bundles instead of IP packets over the network. VDTN architecture categorizes the two logical planes:

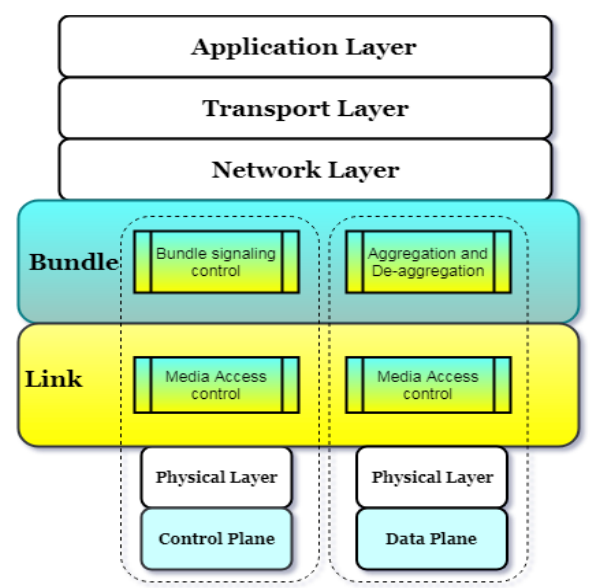

Fig. 2. VDTN Architecture.

1) Control plane.

2) Data plane.

The control plane uses short bandwidth, less-power, and vast-range link, as it continuously assists to let node discovery. It is responsible for a signaling message, node localization, resource reservation and routing of data bundles from the sender to the receiver node. The data plane utilizes excessive bandwidth with high-power and short-range link and its activation take place at estimated time duration, otherwise, the connection of the data plane is not activated. This approach is considered vital because it is significant for energy-constrained vehicular nodes in the network such as stationary nodes as well as guarantees the optimization of the available resources of the data plane. It is responsible for traffic classification, buffer management, scheduling, forwarding bundles among each other and data aggression and de-aggression.

Bundle layer in VDTN is logically divided into two sublayers which are also illustrated in the above figure:

\section{a) Bundle Signaling Control (BSC)}

b) Bundle Aggregation and De-aggregation (BAD)

Bundle signaling control executes the functions of the control plane such as routing, signaling message, and message exchanging and resource reservation. Bundle aggregation and de-aggregation execute the functionality of the data plane which consists of queuing, storage management and scheduling and traffic classification. VDTN provides a promising environment for velar communication with no network infrastructure.

In VDTN, vehicles act as a moving node to carry the message from source to destination. Different protocols are used that perform the paradigms of the store, carry and forward [12]. The Vehicular Delay-Tolerant Network scenario is elaborated in Fig. 3. Applications of VDTN includes:

1) Notification of traffic jam

2) A weather condition report (rainy, windy, stormy)

3) Road accidents

4) Vehicles collision avoidance

5) Message loss. 


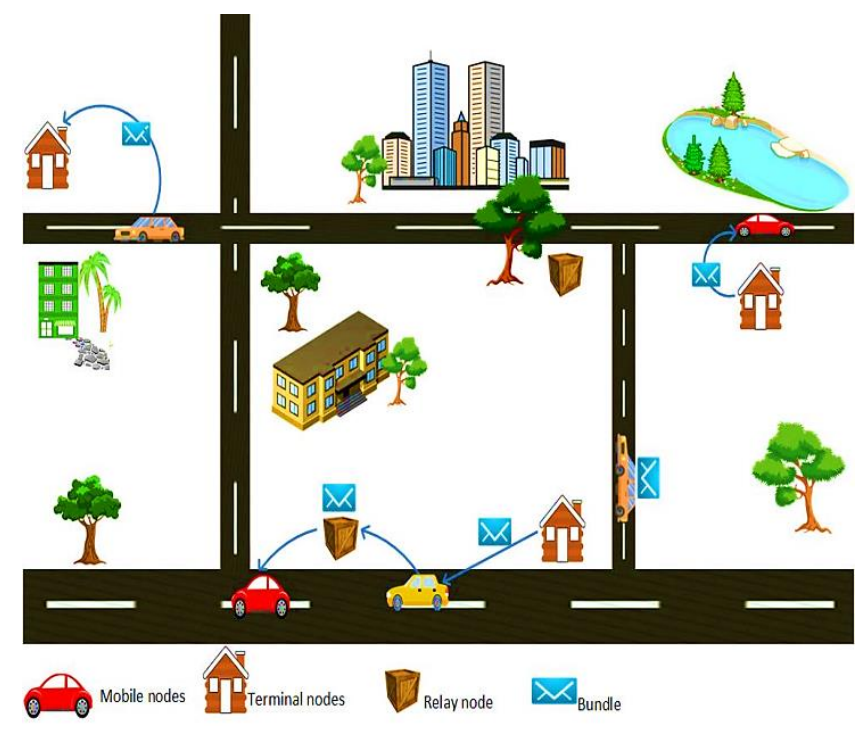

Fig. 3. Scenario1.

Mobile nodes are vigorously allowed to gather and broadcast message bundles through the VDTN. They move along roads and transfer the message bundle that must be conveyed to the terminal nodes. Terminal nodes can be characterized as fixed and mobile nodes. Relay nodes are stationary nodes with capabilities of DTN (store-and-forward) located at road crossings. Mobile nodes interrelate with relay nodes to placing and then picking up message bundles.

\section{LITERATURE REVIEW}

Many routing protocols are published in the literature that carries the data over the network. Here we explain some of the routing protocols and their working so it will be clear how our routing protocol is different and have distinguished features than other available in the literature. Table I elaborates the functionality and some other specifications of routing protocols. The routing protocols are categorized as follow:

MaxProp: (Maximum Priority) [13] is one of the wellknown routing protocols of VDTN. It uses hop count in packets as a measure of network reliability on the way to a short distance. The MaxProp established on the base of store carry and forward paradigms which are usually employed in delay tolerant network. When two vehicles communicate with each other, they exchange the message in a definite order. If the node which is encountered, is the destination node having a message packet then the message is delivered.

Direct Delivery: In the Direct Delivery routing algorithm the message (bundle) is directly sent to the destination nodes [14]. The protocol does not require any information regarding the network so, it is flooding in nature. When the source node generates the message also called bundle then it waits for the destination node to receive it.

Epidemic: It is a flooding-based technique. In Epidemic routing [15] each node replicates its message to every other node until to meet it so far. The message replication is happening after the maintenance of the summary vector. The summary vector is the retained for each individual node that stores the message information which is delivered to the encountered node or stored in its buffer. This flooding base technique wastes the network resources for the sake of message delivery to one destination.

Spray And Wait: Spray And Wait [15] protocol is the advancement of an epidemic protocol. According to this protocol, nodes do not replicate the message to every other node but optimize the number of nodes on which the source node conveys the message. It contains two phases:

1) The Spray Phase

2) The Wait Phase.

In the Spray Phase, the source node performs message replication to the $\mathrm{x}$ nodes and then $\mathrm{x}$ nodes are further spread the message to $\mathrm{y}$ nodes. In the Wait Phase, if a node has a single copied message left then the sender node directly transmits it to the destination node.

ProPHET: This protocol uses an approximation of delivery to conclude the performance measures such as Delivery probability. It uses the matric called Delivery Probability to evaluate the probability of the nodes conveying the message to the destination nodes.

First Contact: A routing protocol in which only a single copy of the message is available over the network. It uses a single path to transfer the message and if the connection is not available then the node stores the data and then transfers it to the first contacted node. It does not delete the message but removes the old messages when there is no space in its buffer.

TABLE. I. ROUTING PRotocols

\begin{tabular}{|c|c|c|c|c|c|}
\hline Routing Protocol & Abbreviation & Estimation Based & Copy & Type & Function \\
\hline Epidemic & Epidemic & No & Unlimited & Blind flooding & Rapid propagation of data \\
\hline SprayAndWait & SnW & No & n_copy & $\begin{array}{l}\text { Controlled } \\
\text { flooding }\end{array}$ & Set the limit to the number of copies \\
\hline ProPHET & Prophet & Yes & Unlimited & Probabilistic & Forward based on encounter history \\
\hline DirectDelivery & $\mathrm{DD}$ & No & Single & Direct & Node moves and delivers the bundle \\
\hline MaxProp & Maxprop & Yes & Unlimited & $\begin{array}{l}\text { Maximum } \\
\text { Probability }\end{array}$ & Delivery likelihood \\
\hline FirstContact & FC & No & Single & Probabilistic & A packet is delivered in the result of random walk \\
\hline DesCom & DC & Yes & Single & Routing Decision & $\begin{array}{l}\text { Decide either packet is sent or not and ensure high } \\
\text { delivery rate }\end{array}$ \\
\hline
\end{tabular}


Author in [16] describes the scheduling and dropping policy of packets that implement traffic planning across the network nodes. According to which, the message is scheduled using one of the following policies: Round Robin, Priority Greedy and Time threshold. According to the dropping policy, the message with the least priority and the lowest remaining TTL is discarded first. The research work [17] describes the influence of nodes in the situation of delivering the message according to which, nodes are categorized as "cooperative", "non-cooperative" and "partly cooperative". Author in [18] illustrates the willingness of cooperating nodes or the ability to participating in message forwarding. Two types of cooperating models are introduced: Upon the reception of message, the node either drop the copy of message packet or retain it in its buffer and follow the rules of the routing algorithm or upon the reception of node, store the copy of message packet in its buffer and forward it to the receiver node. A routing scheme was introduced called Road Accident Prevention (RAP), which introduced the Early Warning to make the promising decision, i.e. chose the alternate route, slow down the speed of the vehicle of change the lane. The paper [19]elaborate buffer management strategy which not only increases delivery rate but also decreases high priority by using the ProPHET routing protocol [20]. According to which, among multiple nearest nodes, the node with maximum delivery probability and situated near to destination is defined as the closest node to the destination due to which delivery rate may be increased. This work [21] describes a message dropping policy which is established on the messages priority and message TTL. According to this policy, the message having the lowest-priority and minimum message TTL are deleted first [22]. Some protocols are also available which specifies the Global Positioning System GPS [23]which are intended to use in the situation where communication is based on intermittent connection, continuous link disconnection or reconnection. Author in [24] provides a congestion control solution to optimize the message delivery probability and reduce the chance of message loss in VDTN and improve the awareness for the drivers of the network on the road. This work [25][26] defines the misbehaving of nodes in terms of the number of copies, such as single, $\mathrm{n}$ and an unlimited number of copies on the set of VDTN and defer the forwarding matric due to which delivery rate is reduced. Author in [6] introduces a parameter named connection_time which can be calculated when the connection between nodes gets started and terminated.

\section{METHODOLOGY}

Vehicles travel in urban and rural areas may vary from one another due to their speed such as car travel, having different speeds as compared to heavy road vehicles. Vehicles speed may also vary due to traffic density and everyday timings such as inside the city, traffic density may be higher than outside the city, at office time car traveling speed may be different than night time. A study [27] shows, if two cars communicate with each other via IEEE $802.11 \mathrm{~g}$ passing at $20 \mathrm{Km}$ per hour is around $20 \mathrm{~s}$ and at $40 \mathrm{Km}$ per hour is approximately $15 \mathrm{~s}$ and at $60 \mathrm{Km}$ per hour is almost $11 \mathrm{~s}$. By keeping this in consideration, if TCP is used at $60 \mathrm{Km}$ per hour then the goodput is low, around 4 out of 10 experimentations, no data is relocated to the destination. UDP provides a better result as compared to TCP because it is a connectionless protocol.

According to our previous work[5], estimation time is predicted time which can be measured by the speed of the vehicle and distance covered by the vehicle. Vehicle speed and position can be visualized using ONE Simulator [28]. Routing decisions are conducted using estimation time [29]. The source node generates a message bundle and searches for a node to carry that message bundle. In this work, when the vehicle carries the message bundle for the sake of message transference and establishes a connection with any other node. Both nodes exchange some information with each other like each other speed, remaining buffer size, and distance from each other. Then the estimated time is calculated from this information.

There is some other information. Like, every message has Time to Live (TTL), after this time message will be terminated and will no longer be available. Transmission time is time to deliver the message from one node to another node. This time depends on the link condition and data rate. We assume the link is good with this $(625 \mathrm{Kbps})$ data rate. Now we have 3 times. First is Estimation Time (ET), second is TTL and third is Transmission Time (Tt).

If Estimation time is greater than TTL it means message may expire soon and sending it may not be useful as a greater TTL message. If Transmission Time is greater than Estimation Time again then there is no point to send the message. Let's suppose Message size is $6250 \mathrm{~Kb}$, in the ideal scenario it will take 10 seconds to send the message, but the estimated time is 9 seconds. So preferred is that a smaller message should be chosen. There are some other conditions and scenario which affect communication. The vehicle carries the message bundle and moving on the road than there could be the following possible scenarios for the vehicle traveling on the road:

1) Scenario 1: One vehicle is stationary, and another vehicle is moving. In this case, the moving vehicle consists of a value greater than zero as it contains some positive value and the stationary vehicle has a value equal to zero. Because of this estimation time will be greater comparatively.

2) Scenario 2: Both vehicles are at stationary conditions having a speed equal to zero. Multiple simulations are established but, this kind of scenario is not available in which both vehicles are stationary having communication, this situation can be possible but having very low probability.

3) Scenario 3: Mutually moving vehicles crossing each other. When two interconnecting vehicles are moving in some directions and communicate with each other than there are further sub scenarios which are as follow: direction.

a) When both vehicles are traveling in a similar

b) When two vehicles are crossing each other but in the opposite direction.

c) When two vehicles are crossing over each other at the juncture.

Scenario a: If mutually vehicles are moving in the same direction then it is harder to calculate estimation time because 
if Vehicle 1 is moving with speed $100 \mathrm{~km} / \mathrm{h}$ and Vehicle 2 is moving with speed $45 \mathrm{~km} / \mathrm{h}$ then there are two possibilities:

- We never know when Vehicle 1 overtakes Vehicle 2 due to variations in their speeds.

- Which vehicle takes a turn and when.

Scenario $b$ and $c$ : According to both scenarios, Vehicle 1 and Vehicle 2 are moving toward each other having a speed value greater than zero. In this case, the meeting time of both vehicles is limited as compared to Scenario A but, can be estimated.

\section{RESUlT AND DISCUSSIONS}

\section{A. Simulation Setup}

For visualization of the simulation environment, a java based tool is used named, ONE[28] (Opportunistic Network Environment). ONE simulator consists of a configuration file termed as "default_setting.txt" in which matrices can be altered according to the need. For the simulation environment, we use a small part of the Helsinki city which is a map-based model having the shortest path available as shown in Fig. 4 in which vehicles move to the destination. Table II shows the values used for simulation.

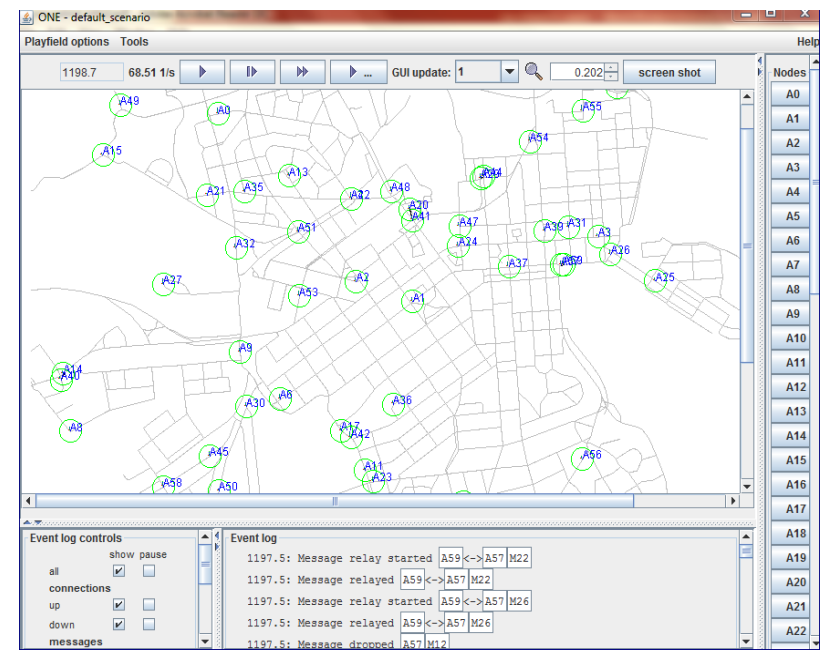

Fig. 4. Simulation Environment.

TABLE. II. SimULATION SETUP

\begin{tabular}{|l|l|l|}
\hline Sr.No & Matrices & Values \\
\hline 1 & sim_time & 21600 \\
\hline 2 & No. of host & $20,40,60$ \\
\hline 3 & Transmit speed & $625 \mathrm{kbps}$ \\
\hline 4 & Transmit range & 60 meters \\
\hline 5 & Buffer_size & $10 \mathrm{M}$ \\
\hline 6 & Vehicle speed & $10-50 \mathrm{~km} / \mathrm{h}$ \\
\hline 7 & Message TTL & 30 \\
\hline 8 & Message size & $100 \mathrm{kB}, 1 \mathrm{MB}$ \\
\hline 9 & World size & 4500,3400 \\
\hline 10 & Wait time & 0,120 \\
\hline
\end{tabular}

\begin{tabular}{|c|c|c|c|c|c|}
\hline Sr.No & $\begin{array}{l}\text { Vehicle } \\
\text { speed }\end{array}$ & Parameter & \multicolumn{3}{|c|}{ No. of Host } \\
\hline & & & 20 & 40 & 60 \\
\hline 1 & \multirow{4}{*}{$10-50$} & $\begin{array}{l}\text { Delivery } \\
\text { Probability }\end{array}$ & 0.8425 & 0.8712 & 0.8712 \\
\hline 2 & & $\begin{array}{l}\text { Average } \\
\text { Buffer-time }\end{array}$ & 297.8803 & 151.0947 & 104.1008 \\
\hline 3 & & $\begin{array}{l}\text { Average } \\
\text { Latency }\end{array}$ & 640.0966 & 453.166 & 415.3612 \\
\hline 4 & & $\begin{array}{l}\text { Overhead } \\
\text { ratio }\end{array}$ & 35.5187 & 148.4858 & 330.2909 \\
\hline
\end{tabular}

\section{B. Simulation Results}

As described earlier, DesCom can conduct the decision, whether the message packet should be sent to the encountered node or find the other suitable node to carry that message packet. Parameters that are excluded are as follows:

1) Delivery probability: It is the relation between the numbers of successfully conveyed messages along with the total number of messages created by the source node.

$D_{S}=$ Successfully delivered messages

Total $_{M}=$ Total number of messages

Delivery Probability $=\frac{D_{S}}{\text { Total }_{M}}$

2) Overhead ratio: Overhead ratio is one of the most vital metrics which shows how competent a protocol is, in terms of precise relay decisions. It indicates the total number of message copies that are relayed in the network before reach to its actual destination.

Relay $_{M}=$ Transmitted messages that are not delivered

Total $_{M}=$ Total number of messages

overhead ratio $=\frac{\text { Relay }_{M}}{\text { Total }_{M}}$

3) Average latency: It is the average time reserved by the messages from the formation to their first delivery at the actual destination node. It is the average time between message generation and received towards the destination node

$D_{T}=$ Time when message is delivered

$C_{T}=$ Time when message is created

Total $_{D}=$ Total number of delivered messages

Average Latency $=\frac{D_{T}-C_{T}}{\text { Total }_{D}}$

4) Buffer time: It is the average time of buffer taken by a vehicle to carry the message packet in its storage. It defines how long a message will remain in the vehicle's buffer. The decrease in average buffer time means that more messages are carried by the encountered vehicles.

TABLE. III. PARAMETER CHECKING WITH DIFFERENT NUMBER OF HOSTS 
So, if the number of nodes is changed then the delivery rate still gave goodput along with low latency and buffer time respectively. Fig. 5, Fig. 6 and Fig. 7 demonstrate the comparison of DesCom with Epidemic, MaxProp, Direct Delivery, First Contact and Prophet Router having parameters that are defined in Table III. In the y-axis, there are values that show the performance of the protocol. In the $\mathrm{x}$-axis, there are predefined parameters in the order of average latency, buffer time average, the number of transported messages and overhead ratio.

From Fig. 5 it can be clearly seen DesCom has the lowest latency and buffer time among all other protocols. While Delivered messages are only less than MaxProp. Here the overhead ratio is also greater than others.

Fig. 6 and Fig. 7 tends to have sort of same trend which is little different than Fig. 5, Here in all of the aspects except overhead ratio, Descom is better than all of the protocols except MaxProp.

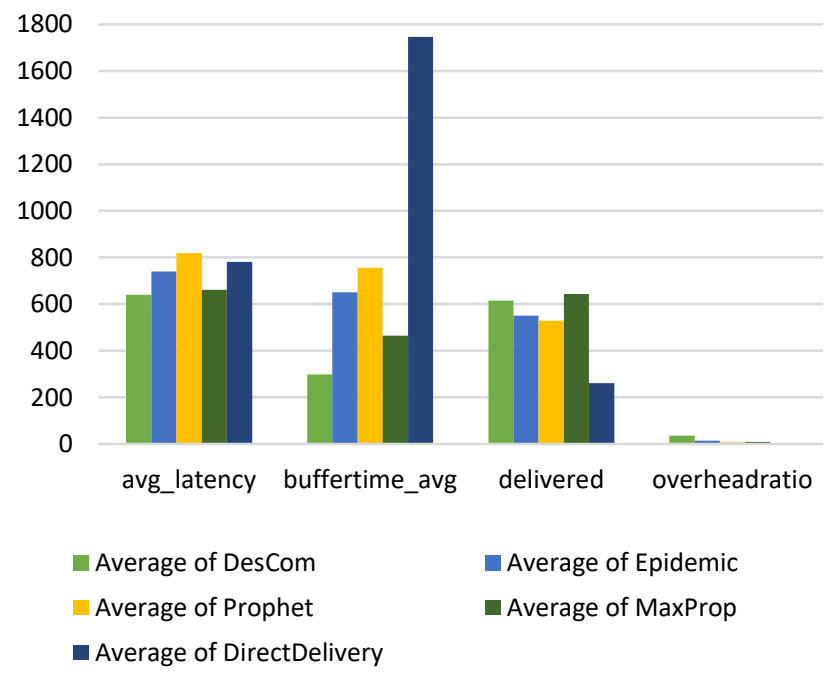

Fig. 5. Comparison with 20 Nodes.

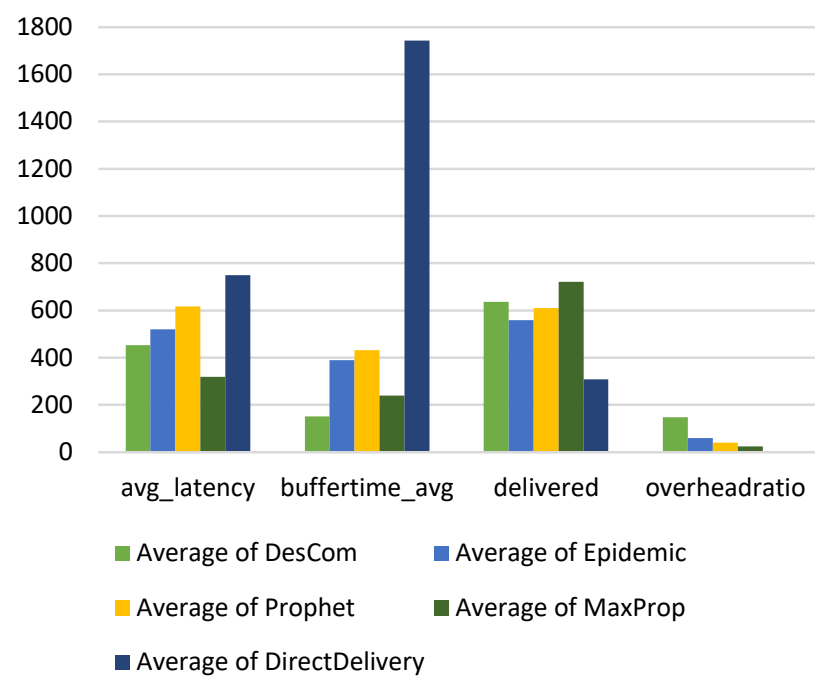

Fig. 6. Comparison with 40 Nodes.

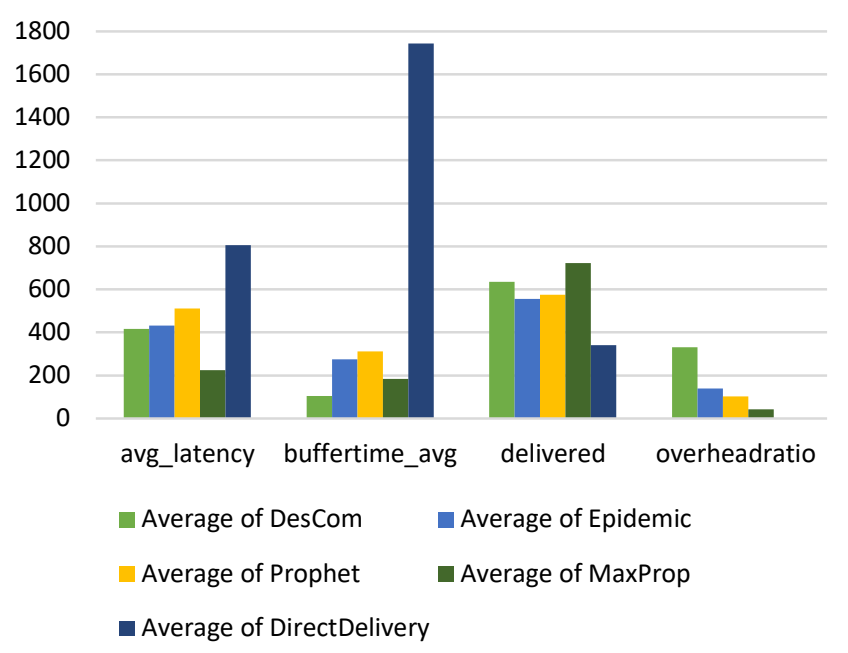

Fig. 7. Comparison with 60 Nodes.

\section{CONCLUSION AND FUTURE WORK}

In this paper based on our previous work we made a routing protocol to prove that estimation time can be used as metric for future routing protocols of such environment where we can calculate the estimation of a connection. In DesCom, routing decisions are conducted using metrics such as TTL, Estimation Time and Transmission Time. Comparisons with other protocols and results show that the routing decisions, taken at the time of message delivery are better which ensures the high delivery rate along with a reduction in buffer time and latency with the increasing number of vehicles. Many simulations were run in ONE simulator with the different number of vehicles to attest that "DesCom" conducts accurate decisions with a variant number of nodes. As Future work, we will suggest and expect more protocols based on estimation time. This estimation time could be a big help to reduce buffer issues. It may also help to reduce average latency and the delivery rate can be increased. We will further work to improve this protocol.

\section{ACKNOWLEDGMENT}

This Research is supported by the National Key Research and Development Program of China (Grant No. 2017YFC0804001) and the National Natural Science Foundation of China (Grant No. 61573328), sponsored by CAS- TWAS President's Fellowship for International Ph.D. Students at School of Computer Science and Technology, University of Science and Technology China.

\section{REFERENCES}

[1] A. Cavalcanti, Elmano \& Rodrigues, Anderson \& Spohn, Marco \& Cézar, Reinaldo \& Fabiano, "VANETs' research over the past decade: Overview, credibility, and trends.," ACM SIGCOMM Comput. Commun. Rev., 2018.

[2] H. W. V. Cerf, S. Burleigh, A. Hooke, L. Torgerson, R. Durst, K. Scott, K. Fall, "Delay-Tolerant Networking Architecture," 2007.

[3] B. Zyl, Ignus \& Rudman, L \& Irwin, "A review of current DNS TTL practices," 2015.

[4] 2018. page 48 - 54. 10.31058/j.ud.2018.12005. Akinyemi, Lateef \& Makanjuola, Tunji \& Shoewu, Oluwagbemiga \& Folorunso, Oluwaseyi. (2018). Smart City and Vehicle Pollution Monitoring Using Wireless Network System. VOLUME 1, "Smart City and Vehicle Pollution 
Monitoring Using Wireless Network System," vol. Vol.1, p. page 48-54, 2018.

[5] A. Ali, M. Shakil, H. Rafique, and S. M. Cheema, "Connection Time Estimation between Nodes in VDTN," Int. J. Adv. Comput. Sci. Appl., vol. 10, no. 1, pp. 339-345, 2019.

[6] A. Ali, N. Sarwar, H. Rafique, I. Hussain, and F. N. Khan, "Connection Time for Routing Decisions in Vehicular Delay Tolerant Network," in Intelligent Technologies and Applications, 2019, pp. 679-690.

[7] K. Keranen, A., OTT, J., "The ONEsimulator for DTN protocol evaluation. In Simutools," in Proceedings of the 2nd International Conference on Simulation Tools and Techniques ,ICST (Institute for Computer Sciences, Social- Informatics and Telecommunications Engineering), 2009, pp. 1-10.

[8] J. 2009 V. N. G. J. Soares, F. Farahmand, and J. J. P. C. Rodrigues, "Improving Vehicular Delay-Tolerant Network Performance with Relay Nodes," 2009 Next Generation Internet Networks, pp. 1-5, "Improving Vehicular Delay-Tolerant Network Performance with Relay Nodes," in Next Generation Internet Networks, 2009, pp. 1-5.

[9] P. R. Pereira, "From Delay-Tolerant Networks to Vehicular DelayTolerant Networks," IEEE Commun. Surv. Tutorials, vol. Vol. 14, no. No. 4, p. Page No.-1166-1182, 2012.

[10] F. F. V. N. G. J. Soares, J. J. P. C. Rodrigues, "Performance assessment of a geographic routing protocol for vehicular delay-tolerant networks," IEEE Wirel. Commun. Netw. Conf., pp. 2526-2531, 2012.

[11] S. Rana, "VDTN Layered Architecture," 2014.

[12] J.-M. Benamar, Maria \& AHNANA, SARA \& ZAHRAE SAIYARI, FATIMA \& Benamar, Nabil \& El Ouadghiri, Driss \& Bonnin, "Study of VDTN routing protocols performances in sparse and dense traffic in the presence of relay nodes," J. Mob. Multimed., 2014.

[13] S. F. A. Shah, M. H. Zafar, I. Andonovic, and T. Jan, "Hybrid routing scheme for Vehicular Delay Tolerant Networks," in 2016 8th Computer Science and Electronic Engineering (CEEC), 2016, pp. 158-163.

[14] R. S. P. Puneet Dwivedi, "Study of Routing Protocols for Delay Tolerant Networks," in International Conference on Contemporary Technological Solutions towards fulfillment of Social Needs, 2018, p. Page -144 .

[15] J. G. F. Antonio S. S. Vieira et al., "VDTN-ToD: Routing Protocol VANET/DTN Based on Trend of Delivery," in The Ninth Advanced International Conference on Telecommunications, 2013, p. pg 135-141.

[16] V. N. G. J. Soares, F. Farahmand, and J. J. P. C. Rodrigues, "Scheduling and drop policies for traffic differentiation on vehicular delay-tolerant networks," SoftCOM 2009 - 17th Int. Conf. Software, Telecommun. Comput. Networks, pp. 353-357, 2009.
[17] D. Benamar, Maria \& Benamar, Nabil \& El Ouadghiri, "The effect of cooperation of nodes on VDTN routing protocols," international Conference on Wireless Networks and Mobile Communications (WINCOM), Marrakech, 2015, pp. 1-7.

[18] X. L. and P. Hui, "An Energy-Efficient n-Epidemic Routing Protocol for Delay Tolerant Networks," in IEEE Fifth International Conference on Networking, Architecture, and Storage, Macau, 2010, pp. 341-347.

[19] K. Yao Théophile, GBALLOU \& Bi, GOORE \& Brou, "Dynamic Weight Dropping Policy for Improve High-Priority Message Delivery Delay in Vehicular Delay-Tolerant Network," Int. J. Adv. Comput. Sci. Appl., vol. Vol.9, no. Issue.10, 2018.

[20] Seung Deok Han and Yun Won Chung, "An Improved PRoPHET Routing Protocol in Delay Tolerant Network," Sci. World J., vol. Volume 201, p. 7 pages, 2015.

[21] A. PENURKAR, Milind et GAJIPARA, "Scheduling and Dropping Policies in Vehicular Delay Tolerant Network," Int. J. Eng. Sci., 2013.

[22] H. Rafique, F. Anwer, A. Shamim, B. Minaei-Bidgoli, M. A. Qureshi, and S. Shamshirband, "Factors Affecting Acceptance of Mobile Library Applications: Structural Equation Model," Libri, vol. 68, no. 2, pp. 99112, 2018.

[23] V. N. G. J. Soares and J. J. P. C. Rodrigues and F. Farahmand, "GeoSpray: A geographic routing protocol for vehicular delay-tolerant networks," Inf. Fusion, vol. 15, pp. 102-113, 2014.

[24] C. O. and M. Radenkovic and U. K. School of computer science, University of Nottingham, "Congestion Aware Spray and Wait Protocol: A Congestion Control Mechanism for the Vehicular Delay Tolerant Network,” Int. J. Comput. Sci. Inf. Technol., vol. Vol, no. No 6, p. pg 83-95, 2015.

[25] P. R. P. and M. P. C. N. Magaia, "Nodes' Misbehavior in Vehicular Delay-Tolerant Networks," in Conference on Future Internet Communications (CFIC), Coimbra, 2013, 2013, pp. 1-9.

[26] A. Ali, H. Rafique, T. Arshad, M. A. Alqarni, S. H. Chauhdary, and A. K. Bashir, "A Fractal-Based Authentication Technique Using Sierpinski Triangles in Smart Devices," Sensors, vol. 19, no. 3, p. 678, 2019.

[27] M. G. R. et Al, "Measuring the capacity of in-car to in-car vehicular networks," IEEE Commun. Mag., vol. 47, no. 11, pp. 128-136, 2009.

[28] P. K. L. B. Er. Richa Thakur, "Delay Tolerant Networks: An Analysis of Routing Protocols with ONE Simulator," in I. J. Computer Network and Information Security, 2016, pp. 51-58.

[29] H. Rafique, A. Shamim, and F. Anwar, "Investigating acceptance of mobile library application with extended technology acceptance model (TAM)," Comput. Educ., p. 103732, 2019. 